\title{
Atrial myxoma: a review of the neurological complications, metastases, and recurrences
}

\author{
A L O NSOL. DESOUSA, J A NS M U L LER, \\ R O B ER T L. C A M P B E L L, S O L O M O N B T N I T Z K Y, \\ A N D L A U R A R A N K I N
}

From the Divisions of Neurological Surgery, Neuropathology, Neuroradiology, and Internal Medicine of Indiana University Medical Center, Indianapolis, Indiana, USA

SUMMARY The neurological complications secondary to embolism from atrial myxoma are reviewed. A patient with intracranial and skeletal metastases is described to emphasise the malignant potentiality of this tumour. A classification of atrial myxoma metastases is presented.

Atrial myxomas are well-known primary tumours of the heart (Prichard, 1951; Heath, 1968; Hardin et al., 1974). Patients usually present in one of three ways: (1) embolic manifestations, (2) cardiac failure, (3) fever, weight loss, malaise accompanied by raised erythrocyte sedimentation rate (ESR), anaemia, and abnormal serum proteins (Greenwood, 1968; Peters et al., 1974). Forty-five per cent of reported cases presented with embolic manifestations, half of them with cerebral involvement (Sylverman et al., 1962; Greenwood, 1968; Peters et al., 1974). Only recently has it been recognised that we face not only local recurrence of the tumour (Gerbode et al., 1967; Bahl et al., 1969; Read et al., 1974; Dang and Hurley, 1976) but distant mycotic aneurysms, caused by tumour invasion of vessel walls, long after the atrial myxoma itself has been removed (New et al., 1970). More recently metastases, presenting as mass lesions, have been reported (Pianov and Kauglova, 1972; Kimbrell and Kaasa, 1973; Read et al., 1974). In reviewing the literature we have found no reported cases with an intracranial metastasis acting as a mass lesion.

\section{Case report}

A 44 year old white woman (SM) was admitted to hospital in May 1976 for evaluation of three months of severe left sided headache and vomiting after an episode of unexplained unconsciousness.

Eight years before admission, she suffered her

Address for reprint requests: Dr Alonso L. DeSousa, Division of Neurosurgery, Indiana University Medical Center, 1100 West Michigan Street, Indianapolis, Indiana 46202, USA.

Accepted 14 June 1978 third stroke in four years characterised by a right hemiplegia and aphasia. The cardiac examination was very suggestive of a left atrial myxoma. The haemoglobin level was $7.2 \mathrm{gm} / \mathrm{dl}$, and she had elevations of the $\alpha-2$ and $\gamma$ globulins, alkaline phosphatase concentration, and ESR. Skeletal radiographs revealed lytic lesions with sclerotic margins in many bones. A large left atrial mass was identified echocardiographically and with cardiac catheterisation. At operation, a $60 \times 30 \times$ $20 \mathrm{~mm}$ myxoma was removed, leaving the interatrial septum intact. A biopsy of the left iliac crest was taken at the site of a lytic lesion; only aseptic necrosis was seen. The hemiplegia improved, and she was discharged.

Four years later she was evaluated for persistent anaemia. The physical examination revealed a systolic ejection murmur, mild residual right spastic hemiparesis, and minimal aphasia. The haemoglobin level was $8.5 \mathrm{gm} / \mathrm{dl}$ and ESR 54 $\mathrm{mm} / \mathrm{hr}$. She still had increased $\gamma$ globulins. The lytic lesions of the skeleton were larger and the borders more sclerotic; the patient declined to undergo another biopsy. A brain scan showed increased blood flow to the right hemisphere and increased uptake of pertechnicate in the right parietal area, corresponding to a cranial lytic lesion. Iron deficiency was identified and therapy begun. She remained unchanged until 1976.

Physical examination showed a lethargic woman. Bilateral papilloedema, a right hemianopia, right upper motor neurone facial weakness, and right spastic hemiplegia were noted. The carotid arteries had good pulsation and were without bruits. A cardiac systolic ejection murmur was audible; no diastolic sounds were heard. The haemoglobin 
level was $12.6 \mathrm{gm} / \mathrm{dl}$ and the ESR $43 \mathrm{~mm} / \mathrm{hr}$. Alpha-2 globulins were slightly increased in the serum. The cardiac silhouette and lung fields were normal radiographically. An echocardiogram failed to reveal recurrence of tumour. The electroencephalogram displayed diffuse delta activity over the left hemisphere. A mass deep within the left temporal area was outlined by pertechnicate brain scan and computerised tomography (Fig. 1). A carotid angiogram demonstrated progressive narrowing of the left internal carotid artery beginning $20 \mathrm{~mm}$ distal to its origin, with complete occlusion just distal to the origin of the ophthalmic artery. The right carotid injection revealed cross filling of the left side and a large avascular left temporal lobe mass shifting the midline structures to the right (Fig. 2). Bone scan showed multiple areas of increased uptake of diphosphonate in the skull, axial skeleton, and long bones which corresponded to the lesions seen radiographically. At operation, an encapsulated mass $(40 \times 30 \times 30 \mathrm{~mm})$ was found protruding into the left lateral ventricle of the brain. The mass originated from a stalk attached to the glomus of the choroid plexus. After complete excision of the mass, there was rapid improvement in the hemiparesis and level of consciousness. At present, though with detectable neurological deficits, she is totally independent.

The $18 \mathrm{~g}$ tumour was smoothly outlined, soft, and spongy. Histologically, there was an intricate system of large, almost sinusoidal blood vessels

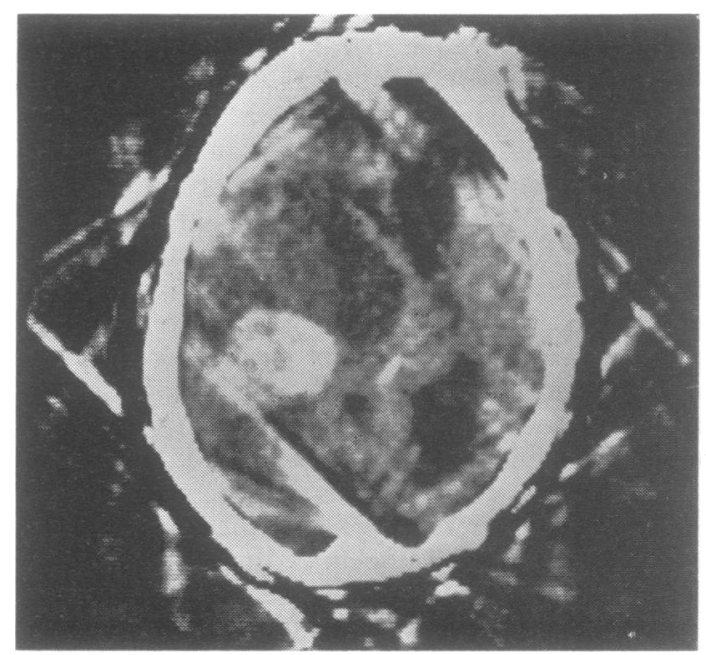

Fig. 1 Computed tomography after intravenous administration of iodinated contrast material showing area of increased density in the left temporoparietal area. Note the enlarged ventricles which are shifted from left to right.

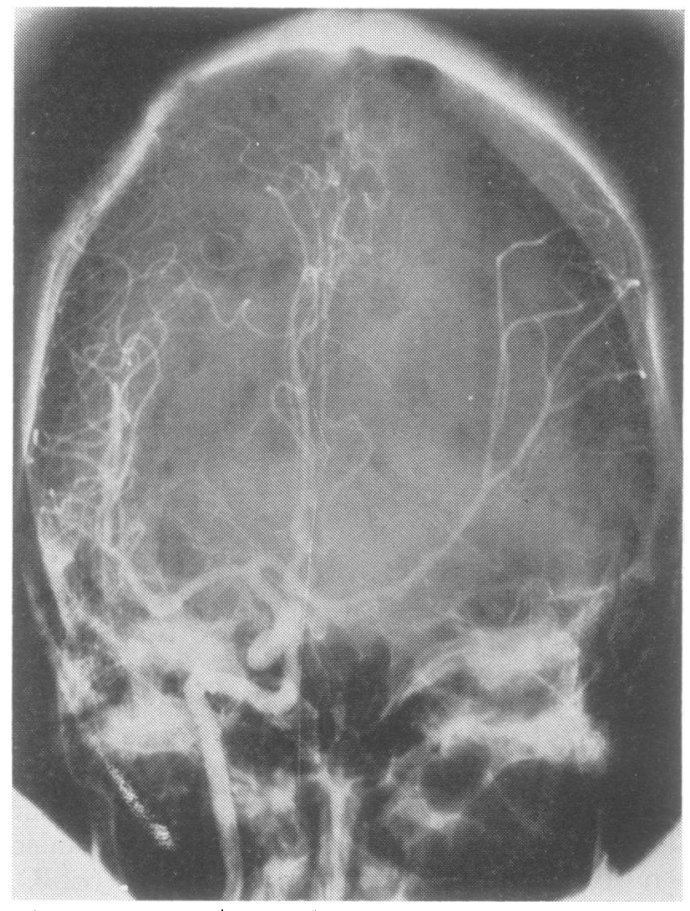

Fig. 2 AP right carotid angiography showing cross filling of the left carotid system with elevation of the left middle cerebral artery indicating a left temporal lobe mass. Also note the lytic lesion in the calvarium.

surrounded by sleeves of myxomatous tissue with occasional connective tissue support (Fig. 3). Masses of faintly staining mucinous material were present. The nuclei were uniformly small and without mitoses.

\section{Discussion}

Our patient had multiple cerebral embolic episodes before the diagnosis of the atrial myxoma. The mass lesion found in the left lateral ventricle was most probably caused by embolisation to the choroid plexus. The location of this metastasis in the choroid plexus, a site generally free of other metastatic tumours, remains baffling.

The bone lesions in our patient have been enlarging since 1968 when they were first noticed (Fig. 4). A bone biopsy sample (iliac crest) in 1968 revealed aseptic necrosis only. It is clear that in the left scapula at least, there is now a large expansile "soap bubble" lesion, evidence of extravascular proliferative growth. Many of the other bone lesions have increased in size as well, surely not to be expected in an infarct.

Although atrial myxomas have been known for 


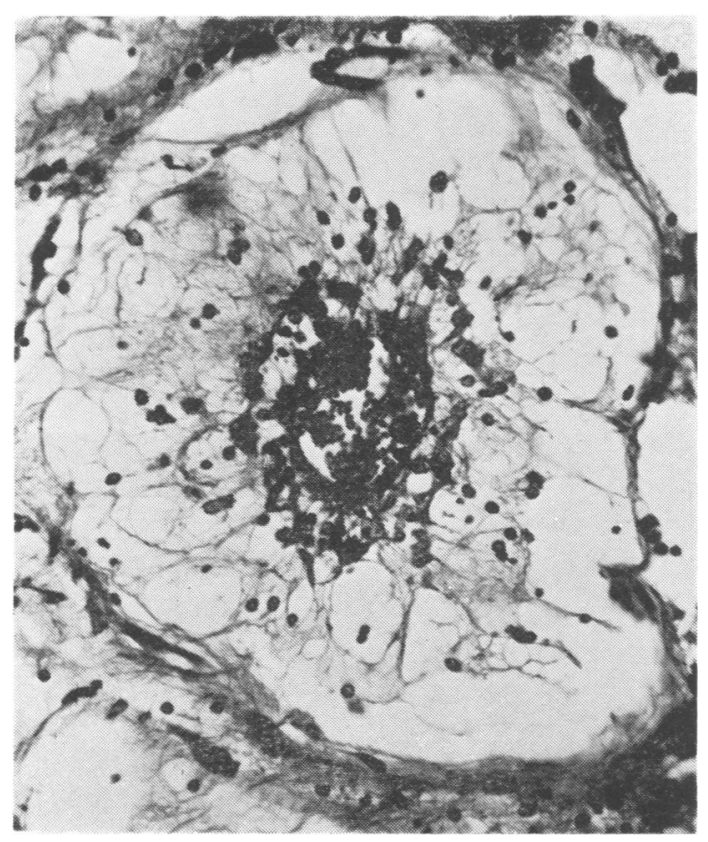

Fig. 3 Blood vessel full of erythrocytes. Neoplastic cells in perivascular crownlike arrangement, embedded in partly precipitated mucinous matrix. $H$ and $E$, original magnification $\times 250$.

many years (Prichard, 1951), it has only recently been recognised that distant metastases may not become evident until many years after removal of the primary lesion (Heath and Mackinnon, 1964; New et al., 1970; Read et al., 1974). All metastases have been haematogenous.

Although a classification of atrial myxoma metastases has its limitations, the following is offered as a working hypothesis:

1. Metastasis, intraluminal only-(a) symptomatic (infarct); (b) asymptomatic.

2. Metastasis with vessel wall invasion-(a) occlusion of vessel, gradual; (b) aneurysm formation.

3. Metastasis with vessel wall transgression-(a) asymptomatic; (b) mass lesion effect.

4. Local cardiac recurrence-(a) at original site; (b) at different site.

\section{SYMPTOMATIC METASTASES}

The incidence of clinically important embolisation from left atrial myxomas may be as high as $45 \%$, with involvement of the brain in half the cases (Sylverman et al., 1962; Greenwood, 1968; Peters et al., 1974). In reviewing the literature, we found 19 patients (Sylverman et al., 1962; Joynt et al., 1965; Burton and Johnston, 1970; Price et al., 1970; New et al., 1970; Yarnell et al., 1971; Stein- metz et al., 1973; Thompson and Simmons, 1974) with demonstrated symptomatic arterial occlusion (necropsy, 14; surgery, 4; both 2) (see Table).

Four patients had extracranial arterial embolisation. Three were operated on for removal of the embolus. One patient had the embolus removed from the right internal carotid artery in the neck with improvement of left hemiparesis. All three patients were discharged, two with mild deficits, and one with complete recovery.

Fifteen patients had cerebral emboli. The neurological deficit was sudden in nine, with hemiplegia or hemiparesis in seven. In the other six cases neurological deterioration was gradual. Neurological symptoms never cleared completely, and all 15 patients with cerebral emboli died. Death was attributed to the neurological disease in 13 cases. The two cases of vertebrobasilar occlusion both underwent necropsy; both also had involvement of the carotid system.

\section{ASYMPTOMATIC METASTASES}

In the same group of patients, other asymptomatic vascular occlusions were seen in 14 (kidney, seven; spleen, four; liver, two; adrenal, one) at necropsy. In one case, a renal biopsy demonstrated an occluded vessel. Bone infarcts after vascular occlusions have been asymptomatic but are typical in radiographs (Kimbrell and Kaasa, 1973; Read et al., 1974). In our patient, the bone lesions have been documented since 1968 (Maroon and Campbell, 1968; Nasser et al., 1972) and still have not become symptomatic.

\section{METASTASES WITH GRADUAL OCCLUSION OF THE} VESSEL

We found two patients with histological proof of vessel wall invasion resulting in narrowing and subsequent occlusion (Price et al., 1970; Steinmetz et al., 1973). The vessel narrowing was mainly caused by intimal thickening by the myxomatous tissue. The narrowing of the vessel and subsequent occlusion were incidental findings at necropsy. Intramural myxomatous tissue without significant change in the lumen was also seen (Price et al., 1970).

METASTASES WITH ANEURYSM FORMATION

Seven patients with mycotic intracranial aneurysms were found (Stoane et al., 1966; Burton and Johnston, 1970; New et al., 1970; Price et al., 1970; Patte et al., 1973; Castaigne et al., 1975; Damasio et al., 1975). The aneurysms were multiple in all patients. In one patient (Castaigne et al., 1975), angiography of the abdominal organs showed two further aneurysms in the "external region of the 


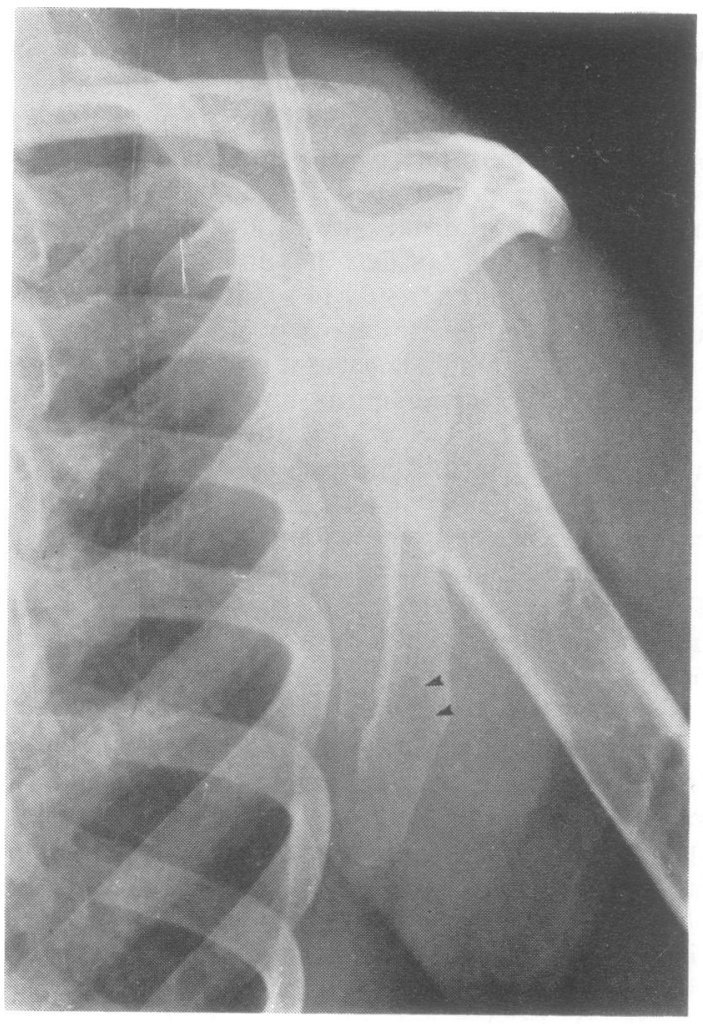

(a)

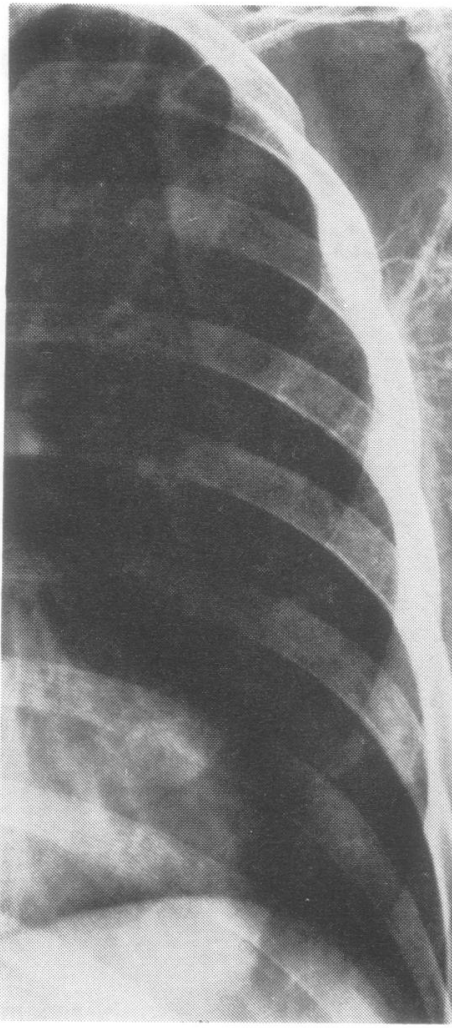

(b)

Fig. 4 Radiographs of left scapula showing marked progression of lytic lesion initially seen in 1968 (arrows) to the large expansile "soap-bubble" lesion seen in 1976 (open arrow head). Also note lytic areas in left humerus (a).

Table Reported cases of arterial embolisation

\begin{tabular}{lcc}
\hline Site & \multicolumn{2}{l}{ Number of cases } \\
\hline Extracranial (4) & 1 & \\
$\quad$ Right cervical internal carotid & 1 & \\
Distal aorta, saddle embolus artery & 1 & \\
Left brachial artery & 1 & \\
Right internal carotid and left brachial & Right & Left \\
\hline Intracranial (15) & 2 & 0 \\
Middle cerebral artery only & 1 & 2 \\
Multiple vessels in 13 (max 3 vessels) & 1 & 3 \\
$\quad$ Intracranial internal carotid & 13 & 6 \\
$\quad$ Anterior cerebral artery & 0 & 2 \\
$\quad$ Middle cerebral artery & 15 & 11 \\
$\quad$ Costerior cerebral artery & & \\
\hline
\end{tabular}

hepatic dome", and multiple aneurysms of the superior polar renal artery. In another case (Price et al., 1970), an aneurysm of the posterior ascending cervical artery was seen angiographically, and many systemic arteries had aneurysms at necropsy. Intracranial aneurysms were seen in both the anterior and posterior circulation. The middle cerebral artery was involved in seven patients, the anterior cerebral artery in four, and the vertebrobasilar system in four.

In one patient (Stoane et al., 1966) repeat angiography two months after removal of the atrial myxoma showed an increase in size of the aneurysms. In another patient (New et al., 1970), there were no neurological symptoms at the time of removal of the atrial myxoma but three years later, when speech difficulty had developed, multiple aneurysms were seen.

Intracranial surgery, with excision of false aneurysms, was carried out in two patients (Burton and Johnston, 1970; Price et al., 1970). There was invasion and proliferation of the myxomatous tissue, replacing the vessel wall. Both patients died in the early postoperative period, and at necropsy both showed similar findings in other vessels.

One patient (Price et al., 1970) presented with an intracranial haemorrhage, possibly the result 
of rupture of such an aneurysm. The haemorrhage involved the lentiform nucleus and internal capsule, with intraventricular and subarachnoid extension at necropsy.

METASTASES WITH VEZSEL WALL TRANSGRESSION Although intraventricular metastasis of this peculiar neoplasm and vessel wall involvement have been known for many years (Ringertz, 1942), actual transgression beyond the vessel has only recently been recognised, perhaps first as microscopic collections of myxoma cells in the leptomeninges (Price et al., 1970).

In 1972, Pianov and Kauglova diagnosed cutaneous masses by biopsy as metastatic myxoma. An additional two patients have been reported with metastatic myxoma, to the vertebral bodies (Kimbrell and Kaasa, 1973) and to multiple bones and soft tissue (Read et al., 1974). No intracerebral cases have been reported.

LOCAL CARDIAC RECURRENCE

Recurrence within the heart was not reported until 1967 (Gerbode et al., 1967). Since then 17 cases with 20 recurrences have been reported (Gerbode et al., 1967; Bahl et al., 1969; Kelly and Bhagmat, 1972; Walton et al., 1972; Maramhao et al., 1973; Read et al., 1974; Jugdutt et al., 1975; Dang and Hurley, 1976). However, results of only a few long-term studies of atrial myxoma cases have been published (Croxson et al., 1972; Hardin et al., 1974; Hedfors and Mogensen, 1974). Reviewing 12 cases of recurrent atrial myxomas, Read et al. (1974) found that the average time to reoperation was only 31 months. It appears that the second lesion grows more rapidly than the primary tumour.

These 17 recurrent cases all had a primary myxoma arising from the septum. Of the 20 recurrences, nine recurred at the interatrial septum at the same site as the original tumour, seven recurred in the same chamber but at different sites, and five were multiple. One case involved the right ventricle of the heart instead (Dang and Hurley, 1976). There were four second recurrences. Fourteen patients who had surgery for recurrences did well after their surgery in short-term follow-up, two died in the postoperative period, and one died three and a half years later from a second recurrence (Jugdutt et al., 1975). The causes for the recurrences had been postulated before (Walton et al., 1972; Read et al., 1974; Dang and Hurley, 1976) and they include known partial excision, inadequate resection (septectomy not performed), implantation of tumour cells, and a growth from a new atrial or ventricular focus.
In the past, there has been controversy as to whether atrial myxomas are in fact true neoplasms, a fact no longer questioned (Ringertz, 1942; Kelly and Bhagmat, 1972; Ferrans and Roberts, 1973; Coltart et al., 1975). The malignant potential of these tumours is not evident in the histology. It cannot be predicted which, if any, of the cardiac myxomas will indeed recur locally, or even give rise to distant metastasis, whether intra- or extravascular. Read et al. (1974) reported a case which demonstrated larger nuclei, prominent nucleoli, and even occasional mitoses in the metastatic tumour.

It is clear, now that more about this enigmatic neoplasm becomes known, that much more care has been taken during cardiac surgery to avoid embolisation, not only because of the embolic phenomenon itself but because viable metastases may become symptomatic many years later, and that the patient with cerebral embolisation is not necessarily cured (Maroon and Campbell, 1968) after the primary tumour is removed.

\section{References}

Bahl, O. P., Oliver, G. C., Ferguson, T. B., Schad, N., and Parker, B. M. (1969). Recurrent left atrial myxoma. Circulation, 40, 673-676.

Burton, C., and Johnston, J. (1970). Multiple cerebral aneurysm and cardiac myxoma. New England Journal of Medicine, 282, 35-36.

Castaigne, P., Laplane, D., Ricou, P. H., and Mallecourt, S. (1975). Multiple intracranial aneurysms of mycotic appearance. Repeated vascular embolic accidents. Myxoma of the left atrium. Revue Neurologique, 135, 339-346.

Coltart, D. J., Billingham, M. E., Popp, L. R., Caves, P. K., Harrison, D. C., and Stinson, E. B. (1975). Left atrial myxoma: diagnosis, treatment and cytological observations. Journal of the American Medical A ssociation, 234, 950-953.

Croxson, R. W., Jewitt, D., Bentall, H. H., Cleland, W. P., Kristinsson, A., and Goodwin, J. F. (1972). Long-term follow-up of atrial myxoma. British Heart Journal, 34, 1018-1023.

Damasio, H., Seabra-Gomes, R., Silva, J. P., DaMasio, A. R., and Antienes, J. B. (1975). Multiple cerebral aneurysms and cardiac myxoma. Archives of Neurology (Chicago), 32, 269-270.

Dang, C. R., and Hurley, E. J. (1976). Contralateral recurrent myxoma of the heart. Annals of Thoracic Surgery, 21, 59-62.

Ferrans, V. J., and Roberts, W. C. (1973). Structural features of cardiac myxomas. Histology, histochemistry and electron microscopy. Human Pathology, 4, 111-146.

Gerbode, F., Kerth, W. J., and Hill, J. D. (1967). Surgical management of tumors of the heart. Surgery, 61, 94-101. 
Greenwood, W. F. (1968). Profile of atrial myxoma. American Journal of Cardiology, 21, 367-375.

Hardin, N. J., Wilson, J. M., Gray, G. F., and Gay, W. A. (1974). Experience with primary tumors of the heart. Clinical and pathological study of seventeen cases. Johns Hopkins Medical Journal, 134, 141-155.

Heath, D. (1968). Pathology of cardiac tumors. American Journal of Cardiology, 21, 315-327.

Heath, D., and Mackinnon, J. (1964). Pulmonary hypertension due to myxoma of the right atrium with special reference to the behavior of emboli of myxoma in the lung. American Heart Journal, 68, 227-235.

Hedfors, E., and Mogensen, L. (1974). Atrial myxoma. Twelve cases operated in Stockholm 1954-1973. European Journal of Cardiology, 211, 101-107.

Joynt, R. J., Zimmerman, G., and Khalifeh, R. (1965). Cerebral emboli from cardiac tumors. Archives of Neurology (Chicago), 12, 84-91.

Jugdutt, B. I., Rossal, R. E., and Sterns, L. P. (1975). An unusual case of recurrent left atrial myxoma. Canadian Medical Association Journal, 112, 1099 1100.

Kelly, M., and Bhagmat, A. G. (1972). Ultrastructural features of a recurrent endothelial myxoma of the left atrium. Archives of Pathology, 93, 219-226.

Kimbrell, D. C., and Kaasa, L. J. (1973). Primary intraluminal aortic myxoma with involvement of several vertebrae. Journal of the American Medical Association, 226, 459-460.

Maramhao, V., Gooch, A. S., Yang, S. S., and Goldberg, H. (1973). Regrowth of a left atrial myxoma. Chest, 63, 98-100.

Maroon, J. C., and Campbell, R. L. (1968). Atrial myxoma: a treatable cause of stroke. Journal of Neurology, Neurosurgery, and Psychiatry, 32, 129133.

Nasser, K. W., Davis, R. H., Dillon, J. C., Tavel, M. E., Helmen, C. H., Feigenbaum, H., and Fisch, C. (1972). Atrial myxoma: phonocardiographic, echocardiographic, hemodynamic, and angiographic features in nine cases. American Heart Journal, 83, 810-824.

New, P. F. J., Price, D. L., and Carter, B. (1970). Cerebral angiography in cardiac myxoma correlation of angiographic and histopathological findings. Radiology, 96, 335-345.
Patte, M., Rakofsky, M., Telerman, M., Struyven, J., Demeurisse, G., and Levi, S. (1973). Left atrium myxoma discovered as the result of a cerebral angiography. Acta Neurologica Belgica, 73, 252-263.

Peters, M. M., Hall, R. J., Cooley, D. A., Leachman, R. D., and Garcia, E. (1974). The clinical syndrome of atrial myxoma. Journal of the American Medical Association, 230, 695-700.

Pianov, R. P., and Kauglova, I. N. (1972). Metastasizing cardiac myxoma. Russian Terapevticheskii Arkhiv, 44, 107-110.

Price, D. L., Harris, J. L., New, P. F. J., and Cantu, R. C. (1970). Cardiac myxoma: a clinicopathologic and angiographic study. Archives of Neurology (Chicago), 23, 558-567.

Prichard, R. W. (1951). Tumors of the heart. Review of the subject and report of one hundred and fifty cases. Archives of Pathology, 51, 98-128.

Read, R. C., White, H. J., Murphy, M. L., Williams, B., Sun, C. N., and Flanagan, W. H. (1974). The malignant potentiality of left atrial myxoma. Journal of Thoracic and Cardiovascular Surgery, 68, 857868.

Ringertz, V. N. (1942). Uber Sog Endokard Myxome. Acta Pathologica Microbiologica Scandinavica, 19, 262.

Steinmetz, E. F., Calanchini, P. R., and Aguilar, M. J. (1973). Left atrial myxoma as a neurological problem: a case report and review. Stroke, 4, 451-458.

Stoane, L., Allen, J. H., and Collins, H. A. (1966). Radiologic observations in cerebral embolization from left heart myxoma. Radiology, 87, 262-266.

Sylverman, J., Olwin, J. S., and Graettinger, J. S. (1962). Cardiac myxomas with systemic embolization. Review of the literature and report of a case. Circulation, 26, 99-102.

Thompson, J. R., and Simmons, C. R. (1974). Arterial embolus. Manifestation of unsuspected myxoma. Journal of the American Medical Association, 228, 864-865.

Walton, J. A., Kahn, D. R., and Willis, P. W. (1972). Recurrence of a left atrial myxoma. American Journal of Cardiology, 29, 872-876.

Yarnell, P. R., Spann, J. F., Dougherty, J., and Mason, D. T. (1971). Episodic central nervous system ischemia of undetermined cause: relation to occult left atrial myxoma. Stroke, 2, 35-40. 